\title{
Acute and Chronic Noradrenergic Effects on Cortical Excitability in Healthy Humans
}

\author{
Hsiao-I Kuo, PhD; Walter Paulus, MD; Giorgi Batsikadze, PhD; Asif Jamil, PhD; \\ Min-Fang Kuo, PhD; Michael A. Nitsche, MD
}

Department of Clinical Neurophysiology, University Medical Center, Georg-August-University, Göttingen, Germany (Ms H.-I. Kuo, Paulus, Mr Jamil, and Nitsche); Department of Psychology and Neurosciences, Leibniz Research Centre for Working Environment and Human Factors, Dortmund, Germany (Ms H.-I. Kuo, Mr Jamil, M.-F. Kuo, and Nitsche); Department of Neurology, University Medical Hospital Bergmannsheil, Bochum, Germany (Dr Nitsche); Department of Neurology, Essen University Hospital, University of Duisburg-Essen, Germany (Dr Batsikadze).

Correspondence: Michael A. Nitsche, MD, Department Psychology and Neuroscience, Leibniz Research Centre for Working Environment and Human Factors, Ardeystrasse 67, Dortmund Germany (nitsche@ifado.de).

\begin{abstract}
Background: Noradrenaline is a major neuromodulator in the central nervous system, and it is involved in the pathophysiology of diverse neuropsychiatric diseases. Previous transcranial magnetic stimulation studies suggested that acute application of selective noradrenaline reuptake inhibitors enhances cortical excitability in the human brain. However, other, such like clinical effects, usually require prolonged noradrenaline reuptake inhibitor treatment, which might go along with different physiological effects.

Methods: The purpose of this study was to investigate the acute and chronic effects of the selective noradrenaline reuptake inhibitor reboxetine on cortical excitability in healthy humans in a double-blind, placebo-controlled, randomized crossover study. Sixteen subjects were assessed with different transcranial magnetic stimulation measurements: motor thresholds, input-output curve, short-latency intracortical inhibition and intracortical facilitation, I-wave facilitation, and short-interval afferent inhibition before and after placebo or reboxetine $(8 \mathrm{mg})$ single-dose administration. Afterwards, the same subjects took reboxetine $(8 \mathrm{mg} / \mathrm{d}$ ) consecutively for 21 days. During this period (subjects underwent 2 experimental sessions with identical transcranial magnetic stimulation measures under placebo or reboxetine), transcranial magnetic stimulation measurements were assessed before and after drug intake.

Results: Both single-dose and chronic administration of reboxetine increased cortical excitability; increased the slope of the input-output curve, intracortical facilitation, and I-wave facilitation; but decreased short-latency intracortical inhibition and short-interval afferent inhibition. Moreover, chronic reboxetine showed a larger enhancement of intracortical facilitation and I-wave facilitation compared with single-dose application.

Conclusions: The results show physiological mechanisms of noradrenergic enhancement possibly underlying the functional effects of reboxetine regarding acute and chronic application.
\end{abstract}

Keywords: reboxetine, noradrenaline, cortical excitability, transcranial magnetic stimulation 


\section{Significance Statement}

Acute and chronic enhancement of noradrenergic brain activity via single dose and chronic administration of reboxetine (RBX) increases cortical excitability via enhancement of facilitation and reduction of inhibition. Chronic RBX resulted in larger increase of intracortical facilitation and I-wave facilitation compared with single dose administration. Chronic RBX itself, without an additional acute loading dose, enhances corticospinal excitability and I-wave facilitation compared to placebo medication.

\section{Introduction}

Noradrenaline is a neuromodulator in the central nervous system that regulates various neuropsychological processes (Robinson, 2012; Bhagya et al., 2015). A single dose of the selective noradrenaline reuptake inhibitor (NRI), reboxetine (RBX), can improve working memory and motor learning in healthy subjects as well as in clinical populations such as depression and stroke (Ferguson et al., 2003; Plewnia et al., 2004; Wang et al., 2009, 2011). The physiological foundation for these effects might be the impact of noradrenaline on neuroplasticity and cortical excitability. Animal studies have shown that noradrenaline enhances long-term potentiation (LTP) as well as long-term depression (Kirwood et al., 1999; Nakadate et al., 2006; Tully et al., 2007). The direction of LTP and long-term depression depends on the activation of $\alpha$ - and $\beta$-adrenoreceptors. Typically, activation of $\beta$-adrenoreceptors enhances LTP, whereas the activation of $\alpha$-adrenoreceptors reduces it (Kemp and Manahan-Vaughan, 2008; Marzo et al., 2009). These receptors also affect various intracellular processes such as ion channel opening via modulation of N-methyl-D-aspartate (NMDA) and gamma-aminobutyric acid (GABA) receptors (Hu et al., 2007; Tully et al., 2007).

Recently, transcranial magnetic stimulation (TMS) measures have been applied to test the effects of noradrenaline on cortical excitability in humans. Corticospinal excitability can be assessed by active and resting motor thresholds (MTs) and the inputoutput curve (I-O curve) (Chen, 2000; Abbruzzese and Trompetto, 2002). MTs reflect neuronal membrane excitability and depend primarily on ion channel activity, as they are increased by voltage-gated sodium channel blockers, but not affected by drugs modulating GABAergic or glutamatergic transmission (Ziemann et al., 1996, 1998a). The I-O curve serves as an index of excitability of larger neuronal populations compared with MTs (Chen, 2000; Abbruzzese and Trompetto, 2002). The I-O curve depends on neuronal membrane excitability, because its slope is decreased by sodium and calcium channel blockers. Furthermore, synaptic mechanisms are involved as it is modulated by drugs influencing the GABAergic and glutamatergic system (Broojerdi et al., 1999; Di Lazzaro et al., 2003; Paulus et al., 2008). Short-latency intracortical inhibition (SICI) and intracortical facilitation (ICF), and motor cortex indirect waves (I-waves) are studied by paired-pulse TMS. SICI is mainly influenced by glutamate and GABA receptors and based on the induction of inhibitory postsynaptic potentials (Ziemann et al., 1996, 1998a; Liepert et al., 1997). ICF is thought to reflect activity of GABAergic and glutamatergic systems. I-waves are thought to be primarily controlled by GABA-related neuronal circuits (Ziemann et al., 1998a, 1998b). In previous studies, a single dose of RBX enhanced the slope of I-O curve and ICF but showed no effect on MTs and intracortical inhibition (Plewnia et al., 2002, 2004). Herwig et al. (2002) reported increased ICF, but also decreased intracortical inhibition under RBX. With regard to the contribution of adrenergic receptor subtypes, following a single dose of the $\alpha$-2-adrenoreceptor agonist guanfacine, a decrease of the I-O curve and ICF as well as an increase of intracortical inhibition have been described (Korchounov et al., 2003). Thus, the RBX-induced excitability enhancement, which differs relevantly from the effect of $\alpha$-2-adrenoreceptor activation, might be primarily driven by excitatory effects of $\beta$-adrenoreceptors. These findings confirm that noradrenaline is involved in human brain excitability, but the mechanism is complex and probably receptor subtype dependent. The above-mentioned pharmacological TMS studies were conducted via single-dose protocols. In clinical applications, therapeutic effects are usually obtained after some weeks (Kasper et al., 2000). It might be speculated that the physiological effects of the respective substances differ between single dose and chronic treatment. Therefore, we aimed to compare the effects of single dose with chronic treatment of RBX on motor cortex excitability in healthy volunteers, using a variety of single and paired-pulse TMS measures tackling different ion channels and receptors. In accordance with previous studies, we hypothesized that a single dose of RBX enhances cortical excitability in healthy humans. According to the superior clinical results obtained from chronic treatment, we furthermore hypothesized that chronic application might also lead to more pronounced enhancement of cortical excitability compared with a single-dose application.

\section{Methods}

\section{Subjects}

Sixteen healthy subjects ( 8 females) aged $27.5 \pm 4.01$ years (mean $\pm \mathrm{SD}$ ) were recruited. Subjects were all right-handed, between 18 and 50 years old, and currently nonpregnant. None of them had a history of neurological diseases, electric implants in the body, or took other medications during the study period. Written informed consent was obtained from all subjects who participated in the study before inclusion. The study was approved by the Ethics Committee of the University of Göttingen and conformed to the Declaration of Helsinki.

\section{Pharmacological Intervention}

Eight mg reboxetine (RBX) or an equivalent placebo (PLC) drug was administered 2 hours before the start of each experimental session, allowing the verum substance to induce a stable plasma level and prominent effects in the central nervous system (Pellizzoni et al., 1996; Dostert et al., 1997). Steady-state plasma concentrations are achieved after 5 days of drug intake, and clinically, the majority of antidepressants have therapeutic effects after approximately 2 weeks of treatment (Dostert et al., 1997; Kasper et al., 2000). Thus, for the chronic RBX condition, we designated a 3-week period of RBX intervention and started to measure cortical excitability after 2 weeks of application.

\section{Monitoring of Motor Cortical Excitability}

Motor evoked potentials (MEPs) were induced in the right abductor digiti minimi muscle (ADM) by single-pulse TMS over the left primary motor cortex, conducted by a Magstim 200 magnetic stimulator (Magstim Company) with a figure-of-eight magnetic 
coil (diameter of one winding $=70 \mathrm{~mm}$; peak magnetic field $=2.2$ T). For the paired-pulse TMS protocols, the coil was connected to 2 Magstim 200 stimulators via a bistim module. The coil was held tangentially to the skull, with the handle pointing backwards and laterally at $45^{\circ}$ from midline. The optimal position was defined as the site where TMS resulted consistently in the largest MEP. Surface electromyography was recorded from the right $\mathrm{ADM}$ by use of $\mathrm{Ag}-\mathrm{AgCl}$ electrodes in a belly tendon montage. The signals were amplified, and band-pass filtered $(2 \mathrm{~Hz}$ to $2 \mathrm{kHz}$; sampling rate, $5 \mathrm{kHz}$ ). Signals were digitized with a power 1401 data acquisition interface (Cambridge Electronic Design) and stored for offline analysis.

\section{Motor Threshold Determination}

The resting MT (RMT) was defined as the minimum TMS intensity that elicited a peak-to-peak MEP of 50 to $100 \mu \mathrm{V}$ in the relaxed muscle in at least 3 of 6 consecutive trials. The active MT (AMT) was the minimum intensity eliciting a MEP response of $\sim 200$ to $300 \mu \mathrm{V}$ during moderate spontaneous background muscle activity ( $15 \%$ of the maximum muscle strength) in at least 3 of 6 consecutive trials.

\section{I-O Curve}

The I-O curve was determined using TMS intensities of 100, 110, 130, and 150\% RMT (15 stimuli per block [each intensity], with the order of the blocks randomized).

\section{SICI-ICF}

Short-latency intracortical inhibition and facilitation were measured by a TMS paired-pulse protocol including ISIs of 2, 3, 5, 10, and $15 \mathrm{~ms}$. The first 3 ISIs represent inhibitory, and the last 2 ISIs reveal facilitatory effects, which reflect excitability of inhibitory and excitatory interneurons, respectively (Kujirai et al., 1993). In this protocol, the subthreshold conditioning stimulus (determined as $70 \%$ of AMT) precedes the test stimulus. The test pulse was adjusted to achieve a baseline MEP of $\sim 1 \mathrm{mV}$ and readjusted during the respective stimulation protocols, if needed, to compensate for effects of global excitability changes on test-pulse amplitude. The pairs of stimuli were organized in 15 blocks, where each ISI was represented once together with one additional single test pulse in a pseudo-randomized order for each block.

\section{I-Wave Facilitation}

I-wave facilitation was investigated using a TMS paired-pulse protocol including ISIs of 1.1, 1.3, 1.5, 2.3, 2.5, 2.7, 2.9, 4.1, 4.3, and $4.5 \mathrm{~ms}$ (Ziemann et al., 1998b). In this protocol, the TMS test stimulus precedes the conditioning stimulus (determined as $70 \%$ of RMT). The test pulse was adjusted to achieve a baseline MEP of 1 $\mathrm{mV}$ and readjusted during the respective stimulation protocols, if needed, to compensate for effects of global excitability changes on test-pulse amplitude. The pairs of stimuli were organized in blocks in which each ISI and one test pulse was represented once and was pseudo-randomized. These blocks were repeated 15 times.

\section{Short-Interval Afferent Inhibition (SAI)}

SAI combines peripheral and motor cortex stimulation to evaluate activity of the cholinergic systems in the human brain (Di Lazzaro et al., 2006). In this protocol, a suprathreshold electric pulse (width of $200 \mu$ s and an intensity of $200 \%$ of the perceptual threshold) over the ulnar nerve precedes the motor cortex TMS test pulse. The test pulse was adjusted to achieve a baseline MEP of $\sim 1 \mathrm{mV}$ and readjusted during the respective stimulation protocols, if needed, to compensate for effects of global excitability changes on test-pulse amplitude. Peripheral nerve stimulation was delivered by a Digitimer D185 stimulator (Digitimer Ltd). Interstimulus intervals of 20 and 40 milliseconds between the peripheral and cortical stimulus were used (Di Lazzaro et al., 2006). The control-conditioning test pairs and a single TMS pulse control condition were recorded 20 times in random order.

\section{Experimental Procedures}

The study was conducted in a partial crossover design. In the chronic medication condition, we had no participant group that received chronic placebo medication for weeks. The study was divided into 2 parts, each with 2 experimental sessions. Within each part, sessions were carried out in randomized order and separated by at least 1 week. All volunteers completed both parts of the study (4 sessions). The first part ( 2 sessions) of the experiment explored single dose RBX effects. Subjects were seated in a comfortable chair with head and arm rests. The right ADM hotspot was determined over the left primary motor cortex, and 20 MEPs were recorded with the TMS intensity, which elicited on average MEP of $1 \mathrm{mV}$ amplitude $\left(\mathrm{SI}_{1 \mathrm{mV}}\right)$. Afterwards, RMT and AMT were determined using standard procedures. After measuring AMT, a 15-minute break followed to avoid a possible effect of muscle contraction on the next measurements. After this break, the following parameters were recorded in randomized order as baseline measures: I-O curve, SICI-ICF, I-wave facilitation, and SAI. Afterwards, the participants took single-dose placebo (sPLC) or RBX (sRBX). Two hours after medication, TMS was readjusted to obtain single test pulse amplitudes of $1 \mathrm{mV}$, if needed. Then all of the above-mentioned parameters were measured again. The second part (2 sessions) of the experiment explored chronic $\mathrm{RBX}$ effects. The same participants received RBX $(8 \mathrm{mg} / \mathrm{d})$ consecutively for 21 days. During this period, the other 2 sessions with RBX (chronic RBX condition [CRBX]: with RBX at the day of experiment) or placebo (chronic placebo condition [CPLC]: with placebo at the day of experiment) were conducted in randomized order at the end of the second and third week after the start of chronic drug intake. The procedure in each session was the same as in the first part of the study (Figure 1).

\section{Data Analysis}

With regard to the RMT, AMT, and $\mathrm{SI}_{1 \mathrm{mV}}$, the individual means of the TMS intensity at RMT, AMT, and $\mathrm{SI}_{1 \mathrm{mV}}$ were calculated for the before and after drug administration conditions separately. Repeated-measures ANOVA were performed for the above-mentioned data using the RMT, AMT, and $\mathrm{SI}_{1 \mathrm{mV}}$ value as the dependent variable, and drug condition (sPLC, SRBX, CRBX, and CPLC) and time point (pre- and postmedication) as independent withinsubject factors. For significant ANOVA results, exploratory posthoc comparisons were performed using Student's t tests (paired samples, 2 tailed, $P<.05$, not corrected for multiple comparisons).

For the I-O curve, the individual means of MEP amplitudes were calculated for all subjects. Repeated-measures ANOVAs were performed on the above-mentioned data using MEP amplitudes as the dependent variable, and drug condition (sPLC, sRBX, CRBX, and CPLC), time point (pre- and postmedication), and TMS intensity as independent within-subject factors. For significant ANOVA results, exploratory posthoc comparisons 


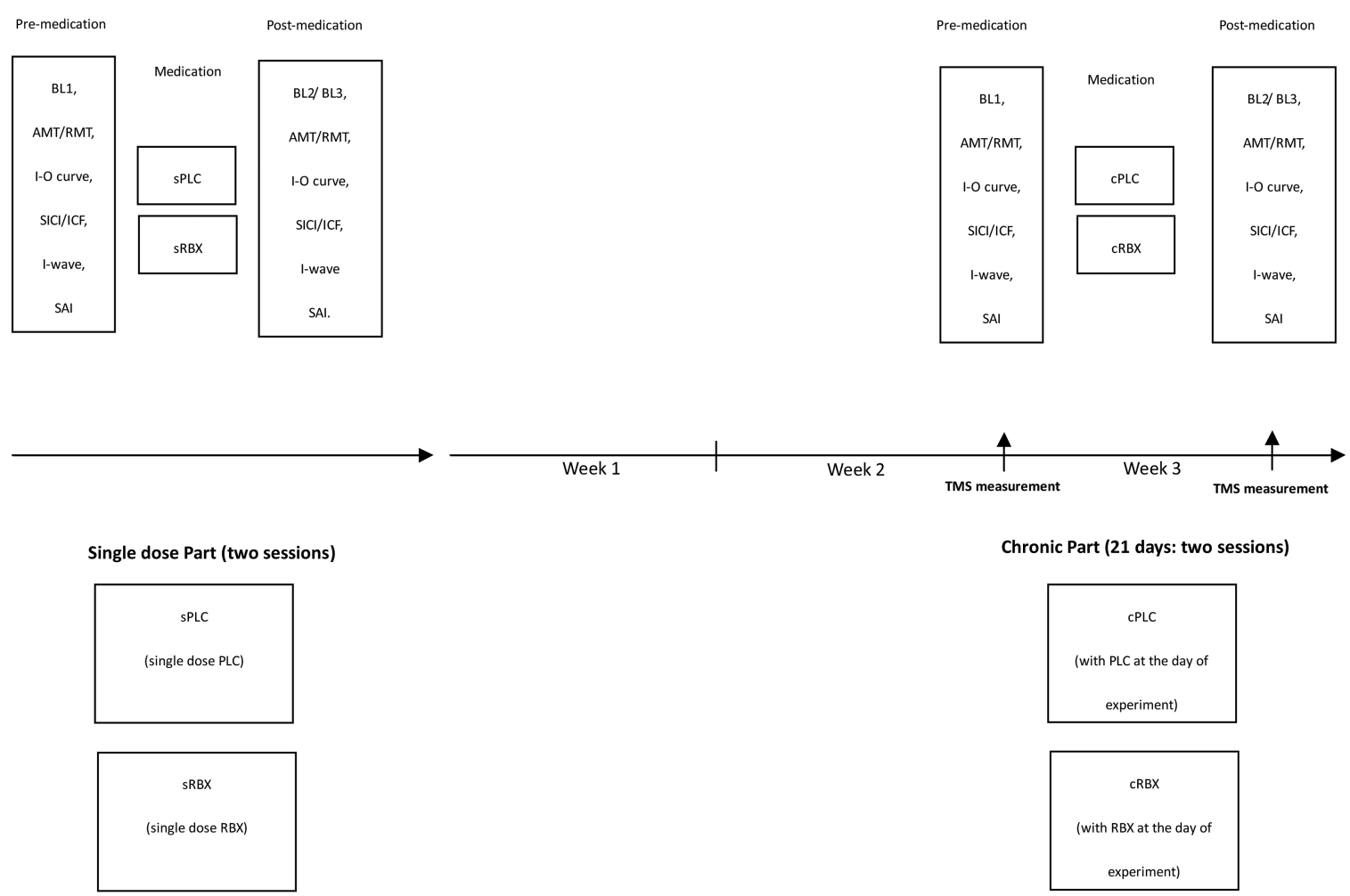

Figure 1. Experimental course of the present study (partial crossover design). The study was conducted in 2 parts (single dose part and chronic part). For the single-dose part ( 2 sessions), subjects received $8 \mathrm{mg}$ single-dose reboxetine (sRBX) or placebo medication (sPLC). First, transcranial magnetic stimulation (TMS) was applied over the left motor cortical representation area of the right abductor digiti minimi muscle (ADM) with an intensity to elicit motor-evoked potentials (MEPs) with a peak-to-peak amplitude of on average $1 \mathrm{mV}\left(\mathrm{SI}_{1 \mathrm{mv}}\right)$. Then, active and resting motor thresholds (AMT and RMT) were determined. Afterwards, input-output curve (I-O curve), shortlatency intracortical inhibition and intracortical facilitation (SICI-ICF), I-wave facilitation, and short-interval afferent inhibition (SAI) were conducted in randomized order. Two hours after intake of the medication, the same TMS measurements were repeated to explore the effect RBX on excitability. For the chronic part (2 sessions), the same subjects took 8 mg RBX consecutively for 21 days. During this period, the other 2 sessions (cRBX: with RBX at the day of experiment or cPLC: with PLC at the day of experiment) were conducted. The design of each session in the chronic part was the same as for the respective single dose condition.

were performed using Student's t tests (paired samples, 2 tailed, $P<.05$, not corrected for multiple comparisons).

Regarding SICI-ICF, I-wave facilitation, and SAI, the mean values were normalized to the respective single-pulse condition. First, intra-individual means were calculated for each condition. To determine significance, repeated-measures ANOVAs were performed (ISIs, drug conditions, and time point as independent within-subject factors and MEP amplitude as the dependent variable). In case of significant results of the ANOVA, exploratory posthoc comparisons were performed using Student's t tests (paired samples, 2 tailed, $P<.05$, not corrected for multiple comparisons).

\section{Results}

In follow-ups, all subjects tolerated TMS and RBX without side effects (one reported a mild tingling sensation during TMS, but did finish the whole session). For RMT, AMT, and $\mathrm{SI}_{1 \mathrm{mV}}$, the respective ANOVAs resulted in no significant main effects of drug and time or the respective interactions (all $P>.05$ ) (Table 1).

\section{I-O Curve}

As displayed in Table 1, the ANOVA showed significant main effects of drug $(F(3)=4.916 ; P=.006)$, intensity $(F(3)=123.338$; $P<.001)$, and drug $x$ intensity interaction $(F(9)=4.869 ; P<.001)$. This is due to enhanced MEP amplitudes caused by increased TMS stimulator output $(100,110,130$, and 150\% RMT) and effects of different drug conditions on MEPs. As shown in Figure 2a, posthoc Student's $t$ tests (paired, 2-tailed, $P<.05$ ) showed significantly larger MEP amplitudes elicited by $130 \%$ and 150\% RMT TMS intensity in the CPLC pre and the CRBX pre condition compared with the sPLC pre condition. Application of single RBX showed significantly larger MEP amplitudes compared with the sPLC post condition in the $130 \%$ and $150 \%$ RMT conditions. Furthermore, CPLC post and CRBX post significantly enhanced the MEP amplitude compared to the sPLC post condition in the $110 \%, 130 \%$, and $150 \%$ RMT TMS conditions, which reflects the long-lasting impact of chronic RBX application on cortical excitability (Figure 2b).

\section{SICI-ICF}

The ANOVA (Table 1) showed significant effects of drug $(F(3)=$ 8.423; $P<.001)$, time $(F(1)=9.489 ; P=.01)$, ISI $(F(4)=5.171 ; P=.002)$, drug $x$ time $(F(3)=13.770 ; P<.001)$, drug $x \operatorname{ISI}(F(12)=4.481 ; P<.001)$, time $x$ ISI $(F(4)=5.831 ; P=.001)$, and drugxtimexISI interaction $(F(12)=5.267 ; P=.001)$. Posthoc Student's t tests (paired, 2-tailed, $P<.05)$ showed that both single-dose and chronic RBX shifted cortical excitability towards an enhancement of excitability. As shown in Figure 3a, sRBX postsignificantly increased facilitation at ISI of 15 milliseconds and decreased inhibition at ISIs of 2, 3, and 5 milliseconds compared with the sPLC postcondition. Administration of chronic RBX resulted in significantly enhanced MEP amplitudes at ISIs of 2, 5, 10, and $15 \mathrm{~ms}$ compared with CPLC post condition (Figure 3b). Moreover, a stronger facilitation at ISIs of 10 and 15 milliseconds and decrease of inhibition at ISIs of 2 and 5 milliseconds was observed under 
Table 1. Repeated-Measures ANOVA Results

\begin{tabular}{|c|c|c|c|c|}
\hline Measurement & Parameters & $\mathrm{df}$ & F-value & $P$ value \\
\hline \multirow[t]{3}{*}{ 1-mV intensity } & Drug & 3 & 0.358 & .784 \\
\hline & Time & 1 & 0.541 & .483 \\
\hline & Drug x time & 3 & 1.583 & .220 \\
\hline \multirow[t]{3}{*}{ AMT } & Drug & 3 & 2.663 & .071 \\
\hline & Time & 1 & 1.583 & .224 \\
\hline & Drug $\mathrm{x}$ time & 3 & 1.254 & .112 \\
\hline \multirow[t]{3}{*}{ RMT } & Drug & 3 & 0.534 & .663 \\
\hline & Time & 1 & 1.254 & .295 \\
\hline & Drug $\mathrm{x}$ time & 3 & 0.544 & .486 \\
\hline \multirow[t]{7}{*}{ I-O curve } & Drug & 3 & 4.916 & .006 \\
\hline & Time & 1 & 3.104 & .104 \\
\hline & Intensity & 3 & 123.338 & $<.001$ \\
\hline & Drug $\mathrm{x}$ time & 3 & 1.762 & .174 \\
\hline & Drug $\mathrm{x}$ intensity & 9 & 4.869 & $<.001$ \\
\hline & Time $\mathrm{x}$ intensity & 3 & 1.230 & .314 \\
\hline & Drug $\mathrm{x}$ time $\mathrm{x}$ intensity & 9 & 1.159 & .330 \\
\hline \multirow[t]{7}{*}{ SICI-ICF } & Drug & 3 & 8.423 & $<.001$ \\
\hline & Time & 1 & 9.489 & .01 \\
\hline & ISI & 4 & 5.171 & .002 \\
\hline & Drug x time & 3 & 13.770 & $<.001$ \\
\hline & Drug x ISI & 12 & 4.481 & $<.001$ \\
\hline & Time $\mathrm{x}$ ISI & 4 & 5.831 & .001 \\
\hline & Drug $\mathrm{x}$ time $\mathrm{x}$ ISI & 12 & 5.267 & .001 \\
\hline \multirow[t]{7}{*}{ I-wave facilitation } & Drug & 3 & 3.254 & .034 \\
\hline & Time & 1 & 2.308 & .157 \\
\hline & ISI & 9 & 14.340 & $<.001$ \\
\hline & Drug $\mathrm{x}$ time & 3 & 1.820 & .163 \\
\hline & Drug x ISI & 27 & 2.233 & $<.001$ \\
\hline & Time $\mathrm{x}$ ISI & 9 & 0.863 & .561 \\
\hline & Drug $\mathrm{x}$ time $\mathrm{x}$ ISI & 27 & 1.767 & .012 \\
\hline \multirow[t]{7}{*}{ SAI } & Drug & 3 & 19.886 & $<.001$ \\
\hline & Time & 1 & 14.218 & .003 \\
\hline & ISI & 1 & 32.029 & $<.001$ \\
\hline & Drug $\mathrm{x}$ time & 3 & 0.650 & .558 \\
\hline & Drug x ISI & 3 & 1.753 & .175 \\
\hline & Time $\mathrm{x}$ ISI & 1 & 1.497 & .247 \\
\hline & Drug $\mathrm{x}$ time $\mathrm{x}$ ISI & 3 & 0.440 & .726 \\
\hline
\end{tabular}

Abbreviations: AMT, active motor threshold; I-O curve, input-output curve; SICI-ICF, short-latency intracortical inhibition and intracortical facilitation; RMT, resting motor threshold; SAI, short-interval afferent inhibition. ${ }^{*}$ Significant results at $P<.05$, d.f., degrees of freedom.

CRBX post compared with sRBX post (Figure 3c). Compared with the SPLC post condition, the CPLC post condition showed a nonsignificant trend towards enhanced facilitation and decreased inhibition (Figure 3d).

\section{I-Wave Facilitation}

The ANOVA (Table 1) revealed significant effects of drug $(F(3)=3.254 ; P=.034)$, ISI $(F(9)=14.340 ; P<.001)$, drug $x$ ISI $(F(27)=2.233 ; P<.001)$, and drug $\mathrm{x}$ time $\mathrm{x}$ ISI interaction $(F(27)=1.767 ; P=.012)$. The results are caused by significant enhancement of I-wave facilitation under both single-dose and chronic RBX conditions. As shown in Figure 4a, application of single-dose RBX significantly enhanced MEP amplitudes at all ISI compared sPLC postcondition. Similarly, CRBX post significantly enhanced I-wave facilitation at nearly all ISIs compared with the cPLC postcondition (Figure 4b). Administration of chronic RBX revealed a significantly enhanced facilitation at nearly all ISIs compared with the SRBX post condition (Figure 4c). In addition, compared with the sPLC post condition, the CPLC post condition demonstrated significantly increased facilitation at all ISI (Figure 4d).

\section{SAI}

The ANOVA revealed significant main effects of drug $(F(3)=19.886 ; P<.001)$, time $(F(1)=14.218 ; P=.003)$, and ISI $(F(1)=32.029 ; P<.001)($ Table 1$)$. Accordingly, after application of single-dose and chronic RBX, SAI was significantly decreased at ISI 20 milliseconds. Compared with the sPLC condition, the cPLC condition demonstrated significantly decreased SAI at ISI 20 milliseconds after drug intake. However, both single-dose and chronic RBX did not have any significant impact on SAI at an ISI of 40 milliseconds (Figure 5).

\section{Discussion}

In the present study, we explored the effects of single-dose RBX and chronic noradrenaline enhancement on cortical excitability in healthy subjects by different TMS protocols (corticospinal excitability: MTs and I-O curves; intracortical excitability: SICIICF, I-wave facilitation, and SAI). In general, our findings show enhanced corticospinal excitability, ICF, and I-wave facilitation, but reduced intracortical inhibition and SAI after single-dose and chronic RBX intake. Furthermore, ICF and I-wave facilitation were more prominently enhanced in the chronic medication condition compared with a single dose. These findings might partially explain why the onset of action of antidepressants takes several weeks in clinical application (Heinbotham and Dunwiddie, 1991; Anderson et al., 2000). Additionally, chronic RBX itself without an additional acute loading dose enhanced facilitation of I-O curve and I-wave facilitation compared with placebo medication, which shows that chronic application of RBX itself results in prolonged excitability alteration.

Regarding corticospinal excitability, AMT, RMT, as well as $\mathrm{SI}_{1 \mathrm{mV}}$ did not differ between single-dose RBX, chronic RBX, and placebo medication. However, I-O curve MEP amplitudes were enhanced under both single-dose and chronic RBX conditions. Animal studies showing that noradrenaline enhances glutamatergic facilitation in the hippocampus might explain these results, as higher TMS intensities of the I-O curve are affected by glutamatergic mechanisms (Hu et al., 2007). Moreover, our results are in line with previous human studies that found that single-dose RBX and the presynaptic $\alpha 2$-antagonist yohimbine increase the slope of I-O curve but do not alter MTs (Plewnia et al., 2001, 2002, 2004). Since measuring the I-O curve involves larger neuronal populations as compared with MTs, the sensitivity of the I-O curve in detecting cortical excitability changes might be superior. This, beyond the impact of glutamatergic mechanisms on the I-O curve, might be another reason why the I-O curves, but not MTs, are modulated by RBX. As the enhancement of the I-O curve originates at cortical or/and subcortical sites, our findings indicate that modulation of central noradrenaline is a possible way to enhance cortico-spinal excitability. Nevertheless, because of the missing 3-way interaction of drug, intensity, and time point, the results of the I-O curve should be interpreted with caution. This missing interaction might be caused by the fact that in the chronic PLC conditions, MEPs were already enhanced because of the impact of chronic RBX intake on cortical excitability, which might have critically diminished the 3-way interaction (drug $\mathrm{x}$ time $\mathrm{x}$ intensity).

SICI-ICF and I-wave facilitation were prominently modulated by RBX, especially in the chronic application mode. Here, we found enhanced ICF and I-wave facilitation as well as a 

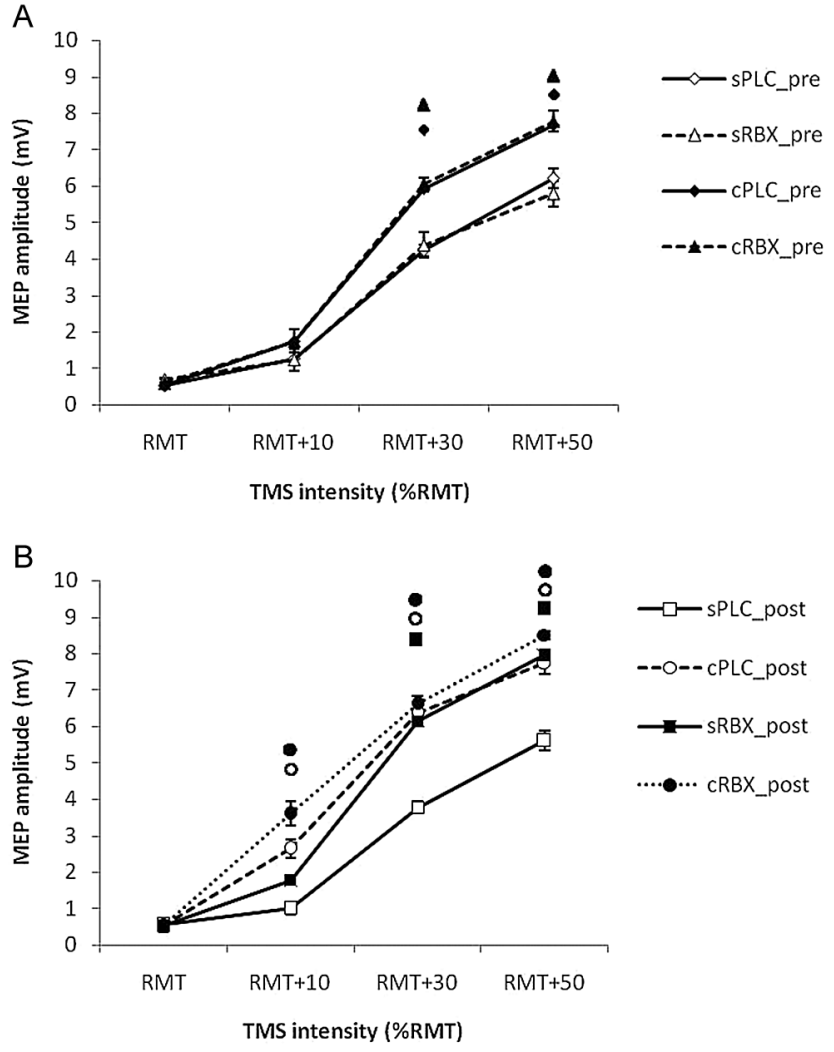

Figure 2. Input-output curve values before and after drug administration. A and B display the MEP amplitudes (means \pm SEM) at $100 \%, 110 \%, 130 \%$, and $150 \%$ of resting motor threshold (RMT) under 4 conditions: single-dose placebo (sPLC), single-dose reboxetine (SRBX), chronic reboxetine + placebo medication at the day of experiment (CPLC), and chronic reboxetine + reboxetine at the day of experiment (cRBX). (A) CPLC pre and CRBX pre showed significantly larger MEP amplitudes compared with sPLC pre elicited by $130 \%$ and $150 \%$ RMT TMS. (B) sRBX post showed significantly larger MEP amplitudes compared with sPLC post at $130 \%$ and $150 \%$ RMT TMS. cPLC post and cRBX post significantly enhanced the MEP amplitude compared with sPLC post at $110 \%, 130 \%$, and $150 \%$ RMT TMS. Floating symbols ( $\bullet$ : cPLC pre; $\mathbf{\Delta}$ : cRBX pre; $\boldsymbol{\bullet}$ : sRBX post; o: cPLC post; $\bullet$ :cRBX post) indicate a significant difference between respective conditions and single placebo condition at the same TMS intensity (Student's $t$ test, 2-tailed, paired samples, $P<.05)$. Vertical bars depict SEM.

decreased inhibition after RBX administration. ICF is regulated predominantly by glutamate receptors with some $\mathrm{GABA}_{\mathrm{A}}$ receptor contribution (Ziemann et al., 1995, 1998a, 1998b). SICI and I-wave facilitation are suggested to be primarily controlled by GABAA receptors (Ziemann et al., 1998b; Ilic et al., 2002; Paulus et al., 2008). Our results thus imply that noradrenaline exerts modulation effects on both excitatory glutamatergic neurotransmission and GABA-related inhibition. These results are in accordance with those of former in vivo and in vitro studies showing that noradrenaline suppresses GABAergic inhibition and enhances glutamatergic facilitation (Hu et al., 2007; Tully et al., 2007). Furthermore, studies in humans have shown that single-dose RBX decreases SICI and increases ICF (Herwig et al., 2002; Plewnia et al., 2002, 2004). These findings, however, contrast with one study, in which application of RBX for 16 days did not affect ICI and ICF (Lange and Liepert, 2007). A possible explanation might be the longer duration of drug intake in our study, which might result in more prominent effects.

Since SAI is directly controlled by the cholinergic system, modulation of the noradrenergic system might have decreased SAI by cholinergic activation (Lazzaro et al., 2000). In the visual
A

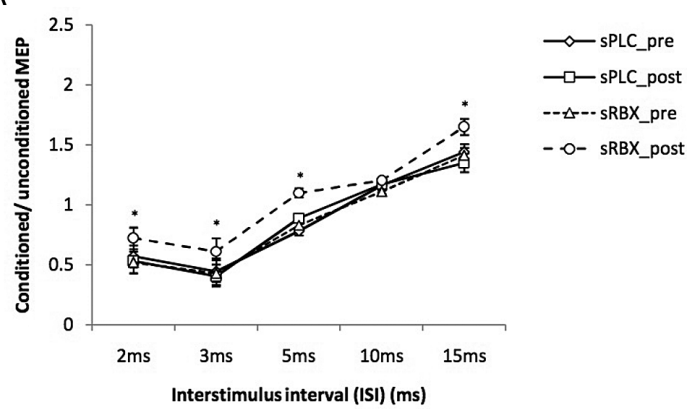

B

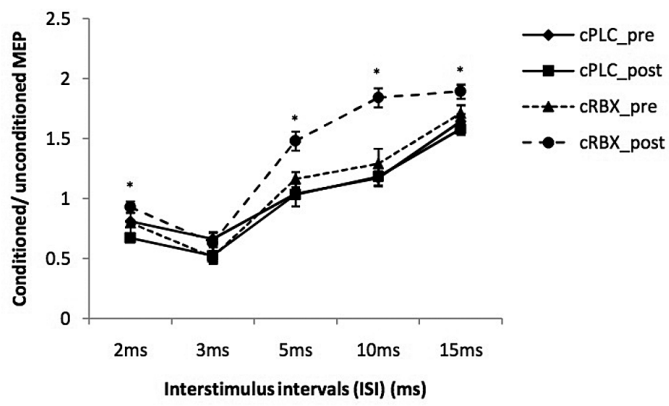

C

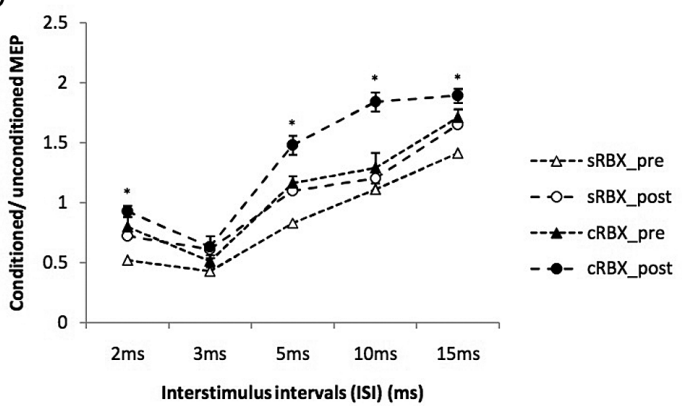

D

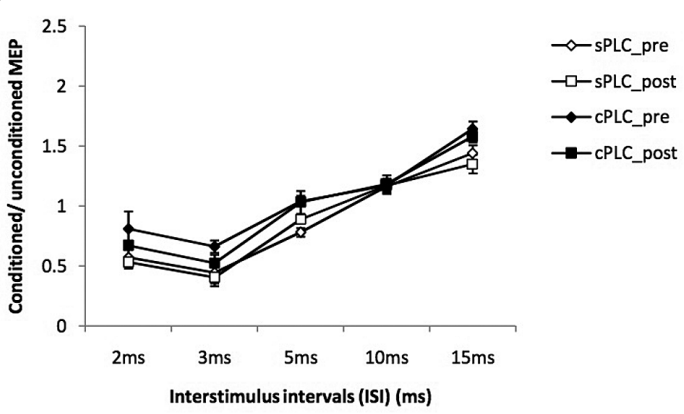

Figure 3. Short-latency intracortical inhibition and intracortical facilitation before and after drug administration. Single-pulse standardized double stimulation MEP amplitude ratios \pm SEM are depicted for ISIs revealing inhibitory (ISIs of 2, 3, and $5 \mathrm{~ms}$ ) and facilitatory (ISIs of 10 and $15 \mathrm{~ms}$ ) effects for different medication conditions: single-dose placebo (sPLC), single-dose RBX (sRBX), chronic reboxetine + placebo medication at the day of experiment (CPLC), and chronic reboxetine + reboxetine at the day of experiment (cRBX). (A) The sRBX post condition significantly increased facilitation for the ISI of 15 milliseconds and decreased inhibition for ISIs of 2, 3, and 5 milliseconds compared with sPLC post condition. (B) CRBX post showed a significant increase of facilitation for the ISIs of 10 and 15 milliseconds and a significant decrease of inhibition for the ISI of 3 and 5 milliseconds compared with CPLC post. (C) CRBX post showed significant facilitation for the ISIs of 10 and 15 milliseconds and significant decrease of inhibition for the ISIs of 2 and 5 milliseconds compared with sRBX post. (D) CPLC post showed a nonsignificant trend towards enhanced facilitation and decreased inhibition compared with sPLC post. Asterisks indicate significant differences (Student's t test, $P<.05$ ). Vertical bars depict SEM. 
A

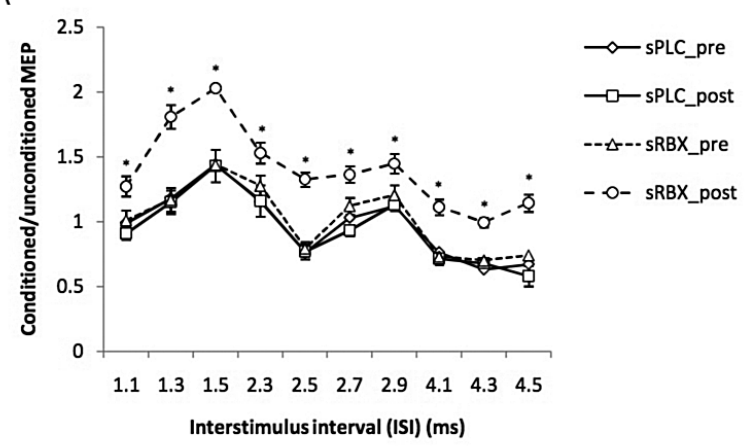

B

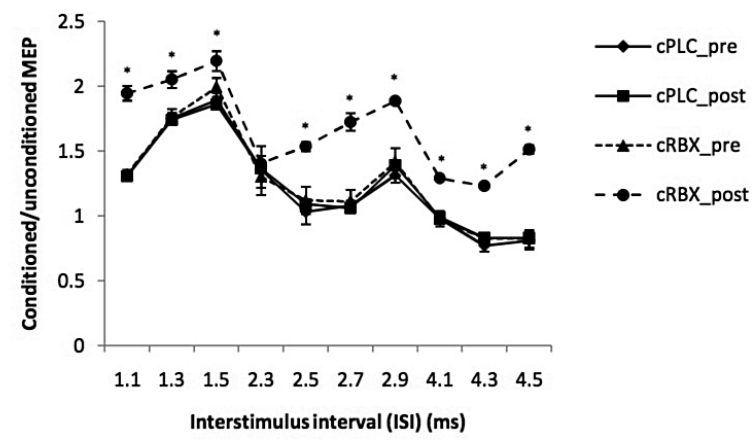

C

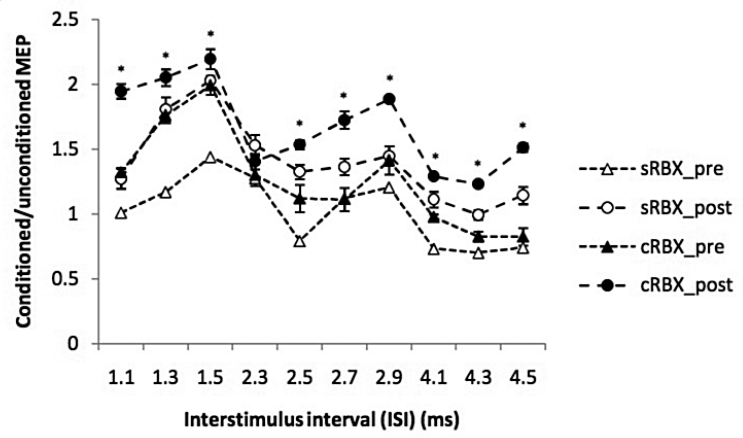

D

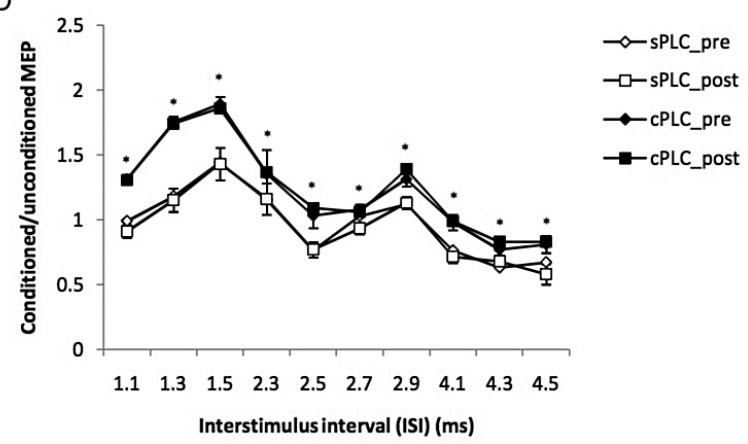

Figure 4. I-wave facilitation before and after drug administration. Single-pulse standardized double stimulation MEP amplitude ratios \pm SEM are depicted for ISIs of 1.1, 1.3, 1.5, 2.3, 2.5, 2.7, 2.9, 4.1, 4.3, and 4.5 milliseconds with different conditions: single-does placebo (sPLC), single-dose reboxetine (sRBX), chronic reboxetine+placebo medication at the day of experiment (CPLC), and chronic reboxetine + reboxetine at the day of experiment (CRBX). (A) sRBX post significantly enhanced MEP amplitude at all ISIs compared with SPLC post. (B) CRBX post significantly enhanced I-wave facilitation at most ISIs compared with CPLC post. (C) CRBX post revealed a significant enhancement of facilitation at most ISIs compared with sRBX post. (D) CPLC post demonstrated significantly increased facilitation at all ISIs compared with sPLC post. Asterisks indicate significant differences (Student's $t$ test, $\mathrm{P}<.05$ ). Vertical bars depict SEM. cortex, slice studies have shown that noradrenaline acts in combination with the cholinergic system, which results in facilitation of LTP (Brocher et al., 1992). However, a contribution of the GABAergic system to the RBX effects cannot be ruled out at present, as SAI is also affected by GABAergic mechanisms (Lazzaro et al., 2007), and other results of our study are in accordance with a prominent impact of RBX on GABA activity.

\section{Mechanisms}

The specific mechanisms responsible for the effects of RBX on cortical excitability in the human brain should be further investigated in forthcoming studies. One possible mechanism is the reduction of potassium conductance by RBX (Hass and Konnerth, 1983). This would result in a depolarization of postsynaptic membranes and enhance calcium influx into the intraneuronal compartment through NMDA receptors and voltage-dependent calcium channels, which might enhance cortical facilitation $(\mathrm{Gu}$, 2002). The $\beta$-adrenoreceptor might be the candidate involved in this mechanism, as activation of $\beta$-adrenoreceptors decreases potassium conductance and results in depolarization of postsynaptic neurons (Hass and Konnerth, 1983). Moreover, the main mechanism suggested by the results of our experiments, an increase of glutamatergic and reduction of GABAergic activity by noradrenergic enhancement, is supported by recent animal studies showing that $\beta$-adrenoreceptors suppress GABAergic inhibition and facilitate activation of NMDA receptors in different brain areas (Hu et al., 2007; Tully et al., 2007).

\section{Functional Relevance}

Recent evidence suggests that cortical excitability and plasticity are disturbed in mood disorders and animal models of stress. RBX serves as a second-line antidepressant. Antidepressant treatment produces opposing physiological effects and thus blocks the effects of stress on performance (Henn and Vollmayer., 2004; Holderbach et al., 2007). However, most of these data so far represent animal studies. Noradrenergic agents have been shown furthermore in pilot studies to improve cognitive functions such as working memory and motor learning in healthy and depressed subjects (Ferguson et al., 2003; Wang et al., 2011). Moreover, a previous study of our group has shown that RBX prolongs and enhances LTP-like plasticity induced by noninvasive brain stimulation (Kuo et al., 2016). LTP is an important physiological derivate of learning and memory formation (Rioult-Pedotti et al., 2000). Deficient LTP and left prefrontal hypo-activity are present in depression and are restituted after antidepressant treatment; thus, respective restitution of brain physiology and reduction of symptoms seem to be associated (Henriques and Davidson., 1991; Rocher et al., 2004; Norman et al., 2007). Taken together, these findings suggest that the enhancement of cortical excitability and LTP-like plasticity may contribute to adaptive changes induced by antidepressant treatment and therefore improve learning and cognition.

Clinically, maximal effects of noradrenergic agents are obtained after weeks or even months of treatment (Kasper et al., 2000), suggesting that longer term adaptive changes might contribute to therapeutic efficacy. Animal studies suggest that both acute and repeated treatments with selective serotonin reuptake inhibitor can enhance synaptic efficacy, but only repeated treatments significantly facilitated the induction of LTP compared with placebo medication (Ohashi et al., 2002). Moreover, brain-derived neurotrophic factor gene expression was downregulated after 4 hours (acute), but upregulated after 14 days 


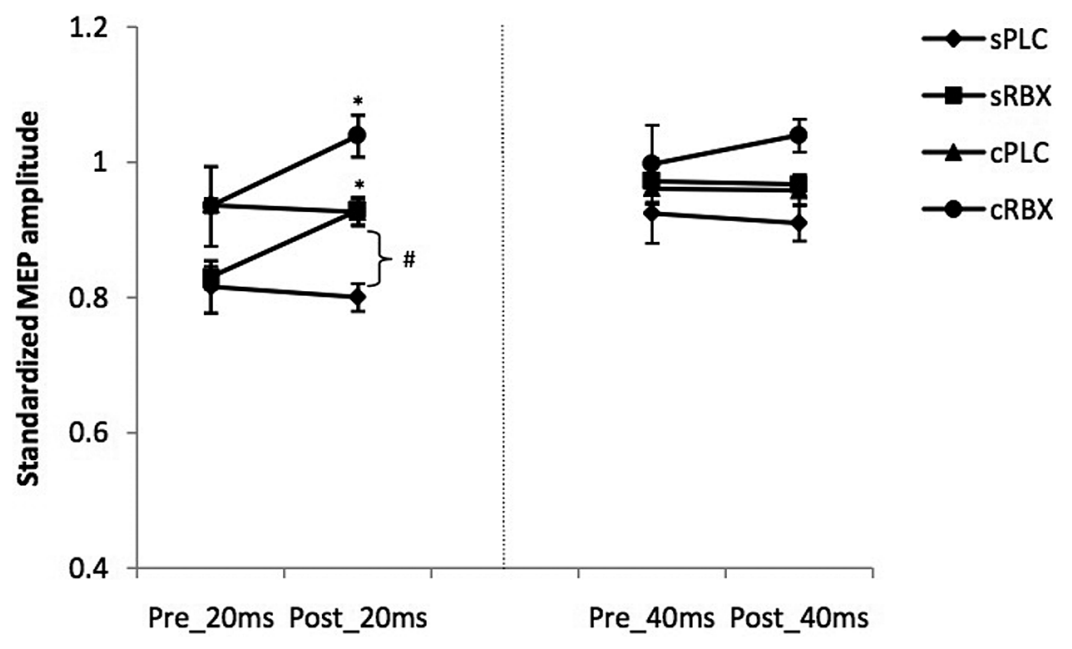

Figure 5. Short-interval afferent inhibition (SAI) before and after drug administration. The figure displays SAI under different conditions: single-dose placebo (sPLC), single-dose reboxetine (RBX), chronic reboxetine + placebo medication at the day of experiment (cPLC), and chronic reboxetine +reboxetine at the day of experiment (cRBX). After application of both, single dose and chronic RBX, SAI was significantly decreased for ISI 20. Compared with the sPLC condition, in the cPLC condition, SAI was significantly reduced at ISI 20 milliseconds. Asterisks indicate significant differences of the MEP amplitude between before and after drug intake (Student's t test, $\mathrm{P}<.05)$. The hash symbol indicates significant differences of the MEP amplitude between sPLC and cPLC after drug intake. Vertical bars depict SEM.

(chronic) of application of selective serotonin reuptake inhibitor in the rat hippocampus (Coppell et al., 2003). Since serotonin and noradrenaline are both central neuromodulators and are effective for treating depression, they might show similar mechanisms regarding acute and chronic effects. In accordance, previous studies suggest that chronic treatment with selective NRI restore learning and memory deficits in an animal model of depression (Bhagya et al, 2015). Furthermore, NRI improved cognitive functions in chronically treated rats (Lapiz et al., 2007). Accordingly, noradrenergic agents improve cognitive function such as working memory and motor learning in healthy and depressed subjects (Ferguson et al., 2003; Wang et al., 2011). These results imply that cortical excitability might be modulated by selective NRI in different ways according to the duration of treatment. It might be speculated that the underlying mechanisms are more prominent noradrenergic enhancement or upregulation of respective receptors. However, based on the current results, it cannot be excluded that the more prominent effects of chronic noradrenaline enhancement are due to enhanced selective NRI concentration in the chronic medication condition, compared with single-dose application. Thus, the specific interaction between dosage, cortical excitability, and noradrenergic activity in the human brain should be explored in more detail in the future.

\section{Limitations}

Some potential limitations of the present study should be taken into account. First, the study was conducted in healthy subjects. In neuropsychiatric diseases, transmitter availability and other features of brain function might be different; future studies are thus needed to explore transferability of these results to patients. In our study, we applied a crossover design in which subjects received a sequence of different experimental sessions. Thus, we did not include a chronic placebo medication condition. There are 2 advantages of this design compared with a parallel group study. First, the influence of confounding covariates is reduced, since each subject serves as his or her own control. In a noncrossover study, even if randomized, it is often the case that different groups are found to be unbalanced on some covariates. Second, a crossover design does require fewer participants than a parallel study, which is more efficient within a limited given time frame. One disadvantage of the crossover design is the missing opportunity to monitor the stability of the obtained excitability parameters over time independent from medication in the chronic medication condition. However, given that respective premedication parameters in the different conditions were quite similar between conditions, we presume the absence of a systematic drift of parameters independent from medication. Another limitation of this study is that it does not allow us to discern if the enhanced effects of chronic medication are due to different physiological mechanisms induced by chronic RBX application, or caused by the higher drug concentration in the chronic medication, compared with single-dose application. The larger effects of chronic medication can indeed be caused at least partially by dose-response effects. Such a dose effect seems plausible, because in the chronic placebo medication condition ("placebo" refers here to the medication at the day of TMS monitoring) we obtained excitability parameters different from no medication conditions. Other effects of chronic medication cannot, however, be ruled out in this study. In future studies, drug concentration measures as well as repetitive TMS monitoring in the chronic medication condition, which was not possible within the current design, would be advantageous. Finally, given the prominent effect of noradrenergic receptor activation on cortical excitability, future studies should explore the relevance of noradrenergic activation on functional outcomes in humans.

\section{Acknowledgments}

This work was supported by the German Federal Ministry of Education and Research -project "Netzwerk psychische Erkrankungen" grant 01EE1403C. Hsiao-I Kuo is supported by a government scholarship, Taiwan. The publication of this article was funded by the Open Access Fund of the Leibniz Association.

\section{Statement of Interest}

Min-Fang Kuo, Hsiao-I Kuo, Asif Jamil, and Giorgi Batsikadze received no financial support or compensation from any individual or corporate entity over the past 3 years for research or professional service and there are no personal financial holdings 
that could be perceived as constituting a potential conflict of interest. W.P. is member of Advisory Boards of GSK, UCB, Desitin. M.A.N. is member of the Advisory Board of Neuroelectrics.

\section{References}

Abbruzzese G, Trompetto C (2002) Clinical and research methods for evaluating cortical excitability. J Clin Neurophysiol 19:307-321.

Anderson I, Nutt D, Deakin J (2000) Evidence-based guidelines for treating depressive disorders with antidepressants: a reversion of the 1993 British Association for Psychopharmacology guidelines. J Psychopharmacology 14:3-20.

Bhagya V, Srikumar B, Traju T, Rao B (2015) The selective noradrenergic reuptake inhibitor reboxetine restores spatial learning deficits, biochemical changes, and hippocampal synaptic plasticity in an animal model of depression. J Neurosci Res 93:104-120.

Brocher S, Artola A, Singer W (1992) Agonist of cholinergic and noradrenergic receptors facilitate synergistically the induction of long-term potentiation in slices of rat visual cortex. Brain Res 573:27-36.

Broojerdi V, Battaglia F, Meullenbacher W, Cohen L (1999) Evaluation of the effects of CNS-active drugs on cortical excitability in intact humans. Neurology 52:457.

Chen R (2000) Studies of human motor physiology with transcranial magnetic stimulation. Muscle Nerve Suppl 9:26-32.

Coppell A, Pei Q Zetterstrom T (2003) Bi-phasic change in BDNF gene expression following antidepressant drug treatment. Neuropharmacology 44:903-910.

Di Lazzaro V, Olivero A, Profice P, Pennisi M, Giovanni S, Zito G, Tonali P, Rothwell JC (2000) Muscarinic receptor blockade has differential effects on the excitability of intracortical circuits in the human motor cortex. Exp Brain Res 135:455-461.

Di Lazzaro V, Oliviero A, Profice P, Pennisi M, Pilato F, Zito G, Dileone M, Nicoletti R, Pasqaletti P, Tonali PA (2003) Ketamine increases human motor cortex excitability to transcranial magnetic stimulation. J Physiol 547:485-496.

Di Lazzaro V, Pilato F, Dileone M, Saturno E, Oliviero A, Marra C, Ranieri F, Quaranta D, Gainotti G, Tonali PA (2006) In vivo cholinergic circuit evaluation in frontotemporal and Alzheimer dementias. Neurology 66:1111-1113.

Di Lazzaro V, Pilato F, Dileone M, Profice P, Ranieri F, Ricci V, Bria P, Tonali PA Ziemann U (2007) Segregating two inhibitory circuits in human motor cortex at the level of $\mathrm{GABA}_{\mathrm{A}}$ receptor subtypes: a TMS study. Clin Neurophysiol 118:2207-2214.

Dostert P, Benedetti M, Poggesi I (1997) Review of the pharmacokinetics and metabolism of reboxetine, a selective noradrenaline reuptake inhibitor. European Neuropsychopharmacology 1:23-35.

Ferguson J, Wesnes K, Schwartz G (2003) Reboxetine versus paroxetine versus placebo: effects on cognitive functioning in depressed patients. International Clinical Psychopharmacology 18:9-14.

Gu Q (2002) Neuromodulatory transmitter systems in the cortex and their role cortical plasticity. Neuroscience 111:815-835.

Hass H, Konnerth A (1983) Histamine and noradrenaline decrease calcium-activated potasium conductance in hippocampal pyramidal cells. Nature 302:432-434.

Heinbotham L, Dunwiddie T (1991) Long-term increases in the evoked population spike in the CA1 region of rat hippocampal pyramidal cells. Nature 302:432-434.

Henriques JB, Davidson RJ (1991) Left frontal hypoactivity in depression. J Abnorm Psychol 100:535-545.
Henn F, Vollmayer B (2004) Basic pathophysiological mechanisms in depression: what are they and how might they affect the course of the illness? Pharmacopsychiatry 37:152-156.

Herwig U, Brauer K, Connemann B, Spitzer M, Schonfeldt-Lecuona C (2002) Intracortical excitability is modulated by a norepinephrine-reuptake inhibitor sa measured with pairedpulse transcranial magnetic stimulation. Psychopharmacology 164:228-232.

Holderbach R, Clark K, Moreu J, Bischofberger J, Norman C (2007) Enhanced long-term synaptic depression in an animal model of depression. Biol Psychiatry 62:373-380.

Hu H, Real E, Takamiya K, Kang M, Ledoux J, Huganir RL, Malinow $R$ (2007) Emotion enhances learning via norepinephrine regulation of AMPA-receptor trafficking. Cell 131:160-173.

Ilic T, Meintschel F, Cleff U, Ruge D, Kessleer K, Ziemann U (2002) Short-interval paired-pulse inhibition and facilitation of human motor cortex: the dimension of stimulation intensity. J Physiol 545:771-782.

Kasper S, Giamal N, Hilger E (2000) Reboxetine: the first selective noradrenaline re-uptake inhibitor. Exp Opin Pharmacother 1:771-782.

Kemp A, Manahan-Vaughan D (2008) Beta-adrenoreceptors comprise a critical element in learning-facilitated long-term plasticity. Cerebral Cortex 18:1326-1334.

Kirwood A, Rozas C, Kirwood J, Perez F, Bert M (1999) Modulation of long-term synaptic depression in visual cortex by acetylcholine and norepinephrine. J Neurosci 19:1599-1609.

Korchounov A, Ilic T, Ziemann U (2003) The alpha 2-adrenergic agonist guanfacine reduces excitability of human motor cortex through disfacilitation and increase of inhibition. Clin Neurophysiol 114:1834-1840.

Kujirai T, Caramia M, Rothwell J, Day B, Thompson P, Ferbert A, Wroe S, Asselman P, Marsden CD (1993) Cortical inhibition in human motor cortex. J Physiol 471:501-519.

Kuo HI, Paulus W, Batskadze G, Jamil A, Kuo MF, Nitsche MA (2016) Acute and chronic effects of nordrenergic enhancement on transcranial direct current stimulation-induced neuroplasticity in humans. J Physiol 7:1-10.

Lange R, Liepert W (2007) Chronic dose effects of reboxetine on motor skill acquisition and cortical excitability. J Neural Transm (Vienna) 114:1085-1089.

Lapiz M, Bondi C, Morilak D (2007) Chronic treatment with desipramine improves cognitive performance of rats in an attentional set-shifting test. Neuropsychopharmacology 32:1000-1010.

Liepert J, Schwenkreis P, Tagenhoff M, Malin J (1997) The glutamate antagonist riluzole suppresses intracortical facilitation. J Neurol Transm 104:1207-1214.

Marzo A, Bai J, Otani S (2009) Neuroplasticity regulation by noradrenaline in mammalian brain. Curr Neuropharmacol 7:286-295.

Nakadate K, Matsukawa M, Okado N (2006) Identification of adrenoreceptor subtype-mediated changes in the density of synapses in the rat visual cortex. Neuroscience 138:37-46.

Norman C, Schmitz D, Furmaier A, Doing C, Bach M (2007) Longterm plasticity of visually evoked potentials in humans is altered in major depression. Biol Psychiatry 62:373-380.

Ohashi S, Matsumoto M, Togashi H, Ueno K, Yoshioka M (2003) The serotonergic modulation of synaptic plasticity in the rat hippocampo-medial prefrontal cortex pathway. Neurosci Lett 342:179-182.

Paulus W, Classen J, Cohen L, Lang C, Lazarro V, Nitsche M, Pascual-Lenone A, Rosenow F, Rothwell JC, Ziemann U (2008) State of the art: pharmacologic effects on cortical excitability 
measures tested by transcranial magnetic stimulation. Brain Stimul 1:151-163.

Pellizzoni C, Poggesi I, Jørgensen N, Edwards D, Paus E, Benedetti M (1996) Pharmacokinetics of reboxetine in healthy volunteers. Single against repeated oral doses and lack of enzymatic alterations. Biopharm Drug Dispos 17:623-633.

Plewnia C, Classen J, Cohen L, Gerloff C (2001) Noradrenergic modulation of human cortical excitability by the presynaptic alpha2-antagonist yohimbine. Neurosci Lett 307:41-44.

Plewnia C, Hoppe J, Heimke C, Bartles M, Cohen L, Gerloff C (2002) Enhancement of human cortico-motoneuronal excitability by the selective norepinephrine reuptake inhibitor reboxetine. Neurosci Lett 330:231-234.

Plewnia C, Hoppe J, Cohen L, Gerloff C (2004) Improved motor skill acquisition after selective stimulation of central norepinephrine. Neurology 62:2124-2126.

Rioult-Pedotti M, Friedman D, Donoghue JP (2000) Learninginduced LTP in neocortex. Science 290:533-536.

Robinson E (2012) Blockade of noradrenaline re-uptake improves accuracy and impulse control in rat performing a five-choice serial reaction time tasks. Psychopharmacology 219:303-312.

Rocher C, Spedding M, Munoz C, Jay TM (2004) Acute stressinduced changes in hippocampal/prefrontal circus in rats: effects of antidepressants. Cerebral Cortex 14:224-229.
Tully K, Li Y, Tsvetkov E, Bolshakov V (2007) Norepinephrine enables the induction of associative long-term potentiation at thalamo-amygdala synapses. Pro Natl Acd Sci USA 104:14146-14150.

Wang L, Fink G, Dafotakis M, Grefkes C (2009) Noradrenergic stimulation and motor performance: different effects of reboxetine on movement kinetics and visuomotor abilities in healthy human subjects. Neuropsychologia 47:1302-1312.

Wang L, Fink G, Diekhoff S, Rehme A, Eickoff S, Grefke C (2011) Noradrenergic enhancement improves motor network connectivity in stroke patients. Ann Neurol 69:375-388.

Ziemann U, Loennecker S, Paulus W (1995) Inhibition of human motor cortex by ethanol. A transcranial magnetic stimulation study. Brain 118:1437-1446.

Ziemann U, Loennecker S, Steinhoff B, Paulus W (1996) Effects of antiepileptic drugs on motor cortex excitability in humans: a transcranial magnetic stimulation study. Ann Neurol 40:367-378

Ziemann U, Chen R, Cohen L, Hallet M (1998a) Dextromethorphane decreases the excitability of the human motor cortex. Neurology 51:1320-1324.

Ziemann U, Tergau F, Wassermann E, Wischer S, Hildebrandt J, Paulus W (1998b) Demonstration of facilitatory I wave interaction in the human motor cortex by paired transcranial magnetic stimulation. J Physiol 511:181-190. 